\title{
Recension: New Suits - Appetite for Disruption in the Legal World
}

\section{Daniel Kraus *}

New Suits - Appetite for Disruption in the Legal World, édité par Michele DeStefano et Guenther Dobrauz est un ouvrage collectif sur l'avenir des professions du droit dans un monde digital. Les quelques 47 auteurs proviennent toutes et tous d'horizons juridiques spécifiques et nous démontrent que, dans chacun de leurs domaines, les mutations à venir sont à nos portes et requièrent un véritable changement d'attitude de la profession.

Citation: $\quad$ Daniel Kraus, Recension: New Suits - Appetite for Disruption in the Legal World, dans: sui-generis 2019, S. 293

URL: $\quad$ sui-generis.ch/110

DOI: $\quad$ https://doi.org/10.21257/sg.110

* Prof. Dr. Daniel Kraus (daniel.kraus@unine.ch), LL.M (Kings College London), titulaire de la chaire de droit de l'innovation, Université de Neuchâtel, associé, Vialex Rechtsanwälte AG, Zurich, fondateur de kraus legal \& ip. 
$1 \quad$ L'ouvrage de plus de 700 pages intitulé: New Suits, Appetite for Disruption in the Legal World, édité par Michele DeStefano et Guenther Dobrauz constitue une lecture fascinante pour toute personne intéressée dans le futur de l'un des derniers domaines qui n'a pas encore été fondamentalement touché par la digitalisation: le droit.

2 La couverture donne déjà le ton, montrant à quoi ressembleront, dans l'imaginaire des éditeurs, les juristes du futur: des (cybers?)-punks en veste de cuir, arborant divers pins de geeks. C'est bien sûr en ouvrant le livre et en commençant à le lire que l'on réalise que tout-e juriste souhaitant voir son entreprise survivre les prochaines années, que ce soit un petit ou un grand cabinet d'avocat-e-s, une entreprise de conseil ou une maison d'édition, devra, plutôt tôt que tard, s'adapter au Nouveau Monde.

Ce volumineux - mais passionnant - ouvrage est divisé en 3 parties, qui répondent à trois questions: la première partie pose la question de savoir pourquoi les juristes ont besoin de nouveaux habits («Why do lawyers need new suits?»); les deux prochaines questions suivent logiquement, et portent sur celle de savoir quels seraient ces nouveaux habits (partie 2: What New Suits might Lawyers Need for the Future?) puis comment les juristes vont pouvoir s'adapter aux nouvelles technologies (partie 3: How will Lawyers fit in the New Suits»). Pour répondre à ces 3 questions, l'ouvrage est subdivisé en plus de 30 chapitres, un prologue, un épilogue et une postface.

4 Le premier chapitre propose une approche historique de l'évolution des ca- binets d'avocats et des prestataires de services juridiques, rappelant que les cabinets d'avocats tels qu'on les connait, avec des associé-e-es, des collaborateurs et des collaboratrices, représentaient une innovation importante dans les années 30. Par la suite, les grandes entreprises ont engagé des conseils internes (in house counsel) pour finalement trouver des alternatives encore plus efficaces consistant à outsourcer leur travail et celui des cabinets d'avocats. Avec l'offshoring, puis avec l'adoption de modes d'engagement flexibles, enfin par la création d'entreprises multidisciplinaires couvrant tous les domaines touchant de près ou de moins près au droit, la profession a subi une mue certes lente, mais ininterrompue. Ce n'est qu'après la crise financière de 2008 et avec le développement des technologies de l'information que de nouveaux partenariats se sont développées avec des grandes entreprises du domaine des logiciels et des start-ups. La frontière entre les prestataires de services traditionnels et alternatifs a ainsi tendance à disparaître. Heureusement, les auteurs ont été prévenants et nous réconfortent d'emblée: les services juridiques demeureront malgré tout fortement dépendantes du capital humain. Mais ils nous avertissent également: les collaboratrices et collaborateurs du domaine du droit devront apprendre à travailler de manière efficace avec les nouvelles technologies et à collaborer de manière interdisciplinaire s'ils veulent apporter de la valeur ajoutée à des industries toujours plus sophistiquées. Cela ne signifie toutefois pas que les valeurs fondamentales du droit doivent être abandonnées dans leur totalité. C'est de ce nouveau cadre juridique dans lequel les fournisseurs de services juridiques vont 
devoir se mouvoir que traite cette ouvrage.

Dans le deuxième chapitre, Mari Sako décrit l'évolution du rôle des general counsel au sein des entreprises. Ce rôle a changé au cours du temps et selon les pays, ce qui n'a pas été sans implications. Comprendre leur rôle de soutien interne aux entreprises qui les emploient, de contrôleur des risques et de partenaires d'affaires est important si les cabinets d'avocats entendent continuer à les assister. L'innovation constitue ainsi une nouvelle discipline clé pour les juristes, laquelle doit être enseignée en tant que telle dans les facultés de droit et autres hautes écoles qui forment les futur-e-s juristes. Selon Michele DeStefano, ces dernier-e-s ne se trouvent certes pas face à un mouvement de disruption tel que celui qui a couté la vie à Kodak, mais dans un mouvement d'innovation adaptative au sens que lui a donné Ron Dolin et Thomas Buley ( $p p$ 83ss, en particulier note 8). L'appel à innover que lance l'auteur vise ainsi tant les grands que les petits cabinets d'avocats et encourage la collaboration accrue pour arriver à offrir des services à $360^{\circ}$ («full-service client service»), proactifs et participatifs, par des personnes qui peuvent analyser les risques des entreprises, parler leur langage et comprendre leur état d'esprit. Bien plus que du conseil, les nouveaux besoins relèvent de la véritable collaboration avec les clients. DeStefano concède que les «deux i» de l'innovation, l'identité et l'individualité, peuvent freiner une telle évolution. En effet, l'identité professionnelle des juristes ne correspond pas encore à l'ADN des innovateurs. Les juristes ont encore trop tendance à vivre en vase clos et à trop peu observer et ques- tionner le monde qui les entoure. En conséquence, plutôt que de véritablement trouver des solutions aux problèmes, ce sont encore les symptômes qui sont traités. Les juristes du futur devront pour leur part être disposés à expérimenter et à travailler de manière transdisciplinaire et collaborative. Il s'agit là d'un véritable changement d'attitude («mindset») qui, selon l'auteur, doit être enseigné dans des cours sur l'innovation aux futurs juristes (p. 107).

6 Que seront alors les métiers juridiques du futur ? Dans le chapitre 4 (Legal Professionals of the Future), John Flood suggère que seront appelées à prospérer les activités dites «à somme nulle» ou les activités distributives, par opposition à celles qui créent des ressources; il s'agirait là notamment des professions liées à la protection de la propriété intellectuelle, à la comptabilité fiscale ou encore à la régulation financière. Or, même ces professions seront peu à peu sujettes à l'automatisation découlant du développement de l'intelligence artificielle (IA).

7 L'avenir des juristes et de leur fonction est donc dépendant de la structure sociale des organisations dans lesquelles leurs activités sont déployées, comme du type de clientèle servie. Les collaborateurs juniors seront les plus menacés, en raison de l'automatisation des tâches les plus simples. Le modèle-même de développement de la profession de l'avocat est ainsi menacé, puisque les cabinets d'avocats sont structurés autour des collaboratrices et des collaborateurs qui deviennent par la suite associés. En même temps, les plus petits bureaux d'avocats seront menacés par le développement des services juridiques en ligne. Alors 
que le besoin de confiance se fera toujours ressentir, la demande d'un nouveau type de généralistes verra le jour, lesquels devront avoir une véritable vue d'ensemble de la «big picture». John Flood rejoint DeStefano en plaidant en faveur d'une évolution radicale des juristes, pour les sortir de leur isolement et s'assurer que les intérêts de la clientèle et de la société soient véritablement servis.

La première partie de l'ouvrage contient également des informations fort utiles sur les nouvelles plates-formes juridiques, appelées à prendre de l'importance à l'avenir, tout particulièrement pour les personnes individuelles et les petites entreprises. Elles les utiliseront pour obtenir des services juridiques rapides et sur mesure. Les fournisseurs de services devront donc à l'avenir s'orienter sur ces nouveaux besoins. (Christoph Küng: Legal Marketplaces and Platforms, pp 132ss). Le domaine de la propriété privée sera également affecté par les nouvelles technologies, à chaque étape des transactions immobilières, en particulier dans l'établissement et l'utilisation du registre foncier et la structuration des transactions, que ce soit par le recours à l'IA pour les due diligence ou à la blockchain pour documenter des transactions (Karl Koller, pp 154ss).

9 Les ressources humaines vont également évoluer dans le domaine du droit. Dans «Gig Economy Lawyers and the Succes of Contingent Workforce Models in Law» (pp 182ss), Marc. O. Morant prévoit que, pour des raisons de coût et de flexibilité, les entreprises seront de plus en plus appelées à acheter les services de spécialistes sur une base occasionnelle. Morant insiste sur la nécessité pour les profes- sions du droit de quitter une attitude qu'il qualifie d'inflexible et de défensive, et d'accepter les nouvelles technologies, les nouvelles structures et modalités d'emploi et les nouveaux modes de résolution des litiges (pp 190ss). Il prédit l'apparition de nouvelles professions telles que celles d'ingénieurs du droit. Les juristes seront rapidement appelés à utiliser les nouveaux outils technologiques tels que le Cloud (une obligation, non plus une option selon Eva Maria Baumgartner, p. 223), à continuer dans l'automatisation de certaines tâches (Michael Grupp et Micha-Manuel Bues), alors que le secteur public devra s'assurer que tant le cadre technique que le cadre juridique permette le développement de solutions de e-government sures (chap. 10, Karl J. Paadam et Priit Martinson: e-Government and e-Justice: Digitalizations of Registers, IDs and Justice Procedures - The Estonia Example).

10 Dans «Lawyer Bots» (pp 270ss), Christian Öhner et Silke Graf considèrent que les Bots peuvent devenir un instrument puissant qui permettra d'améliorer les fonctionnalités juridiques dans une entreprise, le respect de la loi et l'accès à la justice. Mal programmés toutefois, ils risquent d'ajouter des risques d'erreur à la complexité de certaines situations juridiques (p. 285). Heureusement, et malgré le fait que l'industrie du droit se devra d'adopter ces technologies également, les auteurs insistent eux aussi sur le rôle central de l'humain, et donc également des juristes humain-e-s dans la prise de décision.

11 La deuxième partie est consacrée aux habits que les juristes devront revêtir à l'avenir. Les prestataires de services juri- 
diques, de simples fournisseurs de réponse, devront se transformer en de véritables fournisseurs de solutions (Matthias Trummer, Ulf Klebeck et Guenther Dobrauz-Saldapenna: Strategic Mapping of the Legal Value Chain, pp 334ss). Or, de nouveaux service providers apparaissent sur le marché du droit, tels que les maisons d'édition, qui saisissent les opportunités que leur offre la digitalisation, non seulement pour proposer des solutions technologiques pour la gestion des documents, mais également pour la gestion du flux de travail et de l'analyse des données. A terme, les maisons d'édition se présenteront sur le marché comme fournisseurs de véritables services juridiques non seulement en B2B, mais également au client final (Simon Ahammer: Legal Publishers in Times of Digitization of the Legal Market, pp 340ss).

Les métiers du droit devront également enfiler l'habit de la blockchain (Rolf $\mathrm{H}$. Weber: Smart Contracts and What the Blockchain has Got to do With it - (en référence à Tina Turner)) dont on prédit qu'elle aura un impact des plus importants sur la profession, en offrant des nouveaux moyens de garantir la confiance dans des domaines tels que la documentation, les contrats, la datation ou encore la facturation (David Fischer et Pierson Grider: The Blockchain Action in the Legal World). Mais d'autres changements se font déjà percevoir. Ainsi, dans les systèmes qui la connaissent, la procédure du Discovery se transforme gentiment en e-discovery (Juan Crosby, Mike Rowden, Craig Mckeown et Sebastian Ahrens: eDiscovery, pp 388ss). Cet état d'esprit change également au sein des institutions financières, des régulateurs financiers et des fournisseurs de services du domaine, et a donné naissance au «Reg Tech» (David Bundi, pp 40oss). En Suisse notamment, le régulateur y est particulièrement ouvert à l'innovation, tout en précisant que l'innovation ne peut être prescrite par l'État mais doit naître d'une saine concurrence assurée par des conditions cadre favorables, tout en garantissant l'intégrité du secteur financier. L'innovation n'épargne pas non plus d'autres domaines tels que celui de la surveillance des marchés financiers, que Marcel Lötscher affuble du néologisme «Sup Tech» (pp 440ss).

13 Pour pouvoir proposer des solutions, il faut également savoir où chercher. Antonios Koumbarakis propose pour ce faire un chapitre très pratique sur l'avenir de la recherche juridique et passe en revue les plates-formes juridiques au niveau international et en Suisse. Selon l'auteur, la recherche juridique devrait reposer sur quatre piliers, à savoir une recherche précise, qui utilise les dernières technologies, au moyen de plateformes qui permettent à la fois de faire des recherches et de publier, tout en assurant une communication entre différents systèmes. Ces nouveaux modes de recherche devraient également être inclus dans le curricula des études de droit (pp 472ss).

14 L'intelligence artificielle jouera bien évidemment également un rôle important. Toutefois, Luis Ackermann nous avertit: alors que l'assignation à l'IA de tâches laborieuses et intellectuellement peu exigeantes ne posera selon lui pas de problème, tout ce qui ira au-delà risquera de devenir dangereux. Les machines, susceptibles de biais, pourront facilement être manipulées, sans possibilité de réagir de façon sensible à des situations to- 
talement inattendues (pp 482ss). Les exigences en matière de sécurité digitale deviendront donc de plus en plus strictes à l'avenir. L'adoption de systèmes juridiques avancés (Advanced Legal Systems) prendra ainsi du temps et des ressources, mais, malgré cela, l'avantage compétitif qu'une telle technologie apportera sera rémunératrice, en particulier pour les early adopters.

L'automatisation de la création de documents, que Christian Öhner et Silke Graf appellent «des templates sur stéroïdes», prendra également de l'ampleur, même si elle prendra du temps au vu de la culture conservatrice des professions juridiques. Elle présente toutefois de nombreux avantages, notamment en termes de qualité des documents juridiques, de structure en base de données et de gestion effective de contrats, permettant de gagner en efficience. Toutefois, vendus en tant que produits et non plus comme services, ce type de documents met en péril le modèle de tarifs horaires appliqués par les avocats. Cette raison, comme le fait que les avocat-e-s ne sembleraient pas toujours enclin-e-s à partager leurs connaissances, expliqueraient l'absence d'empressement à adopter ces technologies.

La troisième partie de l'ouvrage traite de la façon dont les juristes porteront les nouveaux habits du futur. Maurus Schreyvogel (chap. 23) donne l'exemple des changements opérés au sein de Novartis, de sa perspective de legal counsel. Afin d'améliorer l'efficience des services juridiques, il propose de répondre à trois questions: quelles activités sont-elles fournies? Comment le sont-elles? Comment les optimiser, si nécessaire au moyen de technologies qui pourront sou- tenir le travail de fond des juristes (pp 504ss)? Jordan Urstadt procède à la même analyse, du point de vue de l'avocat cette fois. Il observe lui aussi que les produits remplaceront les services, ce qui aura des implications en particulier sur la fixation des prix et leur distribution (pp 520ss). On assiste ainsi à une véritable passation du pouvoir des cabinets d'avocats aux clients, alors que le domaine du droit était longtemps exempté de procédure de procurement (Silvia Hodges Silverstein et Lena Campagna, pp 530ss). Dans ce cas également, on assiste déjà à une évolution qui consistera non plus, pour les clients, à évaluer le nombre d'heures passées sur un dossier, mais bien les résultats, le niveau des services, l'ensemble des coûts et le temps pris à résoudre le cas. Même le domaine de la restructuration des entreprises en difficulté, des poursuites et des faillites n'est pas épargné, la quantité de données à traiter pouvant plus facilement l'être au moyen de technologies de Big Data. Mais ce domaine pose également la question passionnante des contrats incomplets fondés sur la théorie du prix Nobel d'économie Oliver Hart. Tom Braegelmann (pp 546ss, en particulier 561) évalue l'opportunité de compléter de tels contrats au moyen de «contrats intelligents» (smart contracts), tout en posant clairement les limites de la technologie et en insistant, comme le font d'autres auteurs tout au long de l'ouvrage, sur le rôle central de l'être humain dans ces procédures également.

17 L'évolution n'est toutefois pas uniquement technologique. Elle sera également perceptible dans la façon dont les services juridiques sont offerts, laquelle implique un véritable management des ser- 
vices juridiques et une définition claire des buts, de l'étendue, du calendrier, des ressources humaines et du budget nécessaires à mener à bien un projet juridique. Philipp Rosenauer et Steve Hafner (pp 572ss) nous offrent un excellent résumé des modèles de gestion de dossiers juridiques qui pourront aider en particulier les cabinets d'avocats à demeurer compétitifs à l'avenir. Ces modèles d'affaires ne sont pas épargnés par la culture des hackers, qui tend également à stimuler la créativité dans la résolution de problèmes à l'intersection du droit et des technologies (Jameson Dempsey, Lauren Mack et Phil Weiss, pp 590ss). Tous ces développements mènent ainsi à l'apparition de nouvelles professions du droit et indiquent véritablement à quel point le métier requiert de plus en plus de compétences transversales et technologiques (par ex. CEO d'une entreprise créant des logiciels d'IA juridique), alors que, d'une prestation de services, le métier est en passe de se transformer en une fourniture de produits ( Chief Product Officer»). Noah Waisberg et Will Pangborn (pp 604ss) démontrent pour leur part que les métiers du droit se transforment en véritables professions basées sur l'économie du savoir («Legal Knowledge Engineering Manager»). L'avocat du futur ne correspondra donc pas à celui du passé; il travaillera de manière plus digitale, plus ouverte et plus collaborative (Salvatore Lacangelo, pp 616ss). En conséquence, l'enseignement du droit doit également évoluer. Il faudra inciter les juristes à ne pas craindre d'innover et à travailler en groupe pluridisciplinaire (Michele DeStefano, pp 636ss) ainsi qu'à tenir véritablement compte de la diversité du monde dans lequel nous vivons (Maria Leistner, pp 658ss).
18 On retire de la lecture de ce livre bon nombres d'enseignements. On se remet en question. Faut-il renouveler sa garderobe? Certainement. Cet ouvrage passionnant porte ainsi parfaitement son titre. Le monde du droit vit une mutation qui s'avérera profonde; ceci se vérifiera tout particulièrement dans la pratique de l'avocat-e. Celles et ceux qui ont de l'appétit et qui sont prêt-e-s à se mettre à table seront certainement rassasié-e-s. Celles et ceux qui n'ont pas encore faim, sont encouragé-e- $\mathrm{s}$ à se mettre à table, car l'appétit vient en mangeant. Enfin, celles et ceux qui n'ont pas envie d'y goûter seraient probablement avisé-e-s de changer de restaurant. Quoi qu'il en soit, et malgré l'abondance de technologies auxquelles la profession ne peut échapper, le rôle de l'humain sera toujours fondamental dans la relation de confiance entre l'avocat et ses clients. «New Suits - Appetite for disruption in the Legal World» nous le rappelle à plusieurs reprises, et c'est tout de même réconfortant. Cet excellent ouvrage constitue ainsi une lecture des plus stimulantes; il donne le goût de prendre le virage technologique.

«New Suits - Appetite for Disruption in the Legal World», DeStefano, Michele/Dobrauz-Saldapenna, Guenther, Stämpfli Verlag, 2019.

ISBN: 978-3-7272-1035-8 (édition imprimée, couverture rigide), ISBN: 978-3-7272-1039-6 (livre de poche) 